\title{
Critical study of perturbative approaches to tunneling
}

\author{
E. Tekman \\ Department of Physics, Bilkent University, Bilkent 06533, Ankara, Turkey
}

(Received 31 January 1992)

\begin{abstract}
One of the long-lasting objectives of the theory of tunneling is to express the transmission probability in terms of the wave functions of infinitely separated electrodes. This can be achieved by the application of a perturbative approach to tunneling; in this context the transfer Hamiltonian method has been developed and used. In cases such as scanning tunneling microscopy operating at small tip-sample separation, however, it becomes necessary to go beyond the original transfer Hamiltonian method. In this study we examine the modified forms of the transfer Hamiltonian method using exactly solvable one-dimensional tunneling systems. We find that it is possible to calculate the transmission probability approximately by choosing appropriate boundary conditions for the wave functions used in the transition matrix element expression. However, for low and thin barriers these modified methods still fail to give the correct results. On the other hand, Green's-function techniques which extend the perturbation to all orders yield exact results irrespective of the boundary condition chosen at the interface.
\end{abstract}

\section{INTRODUCTION}

The quest for a generally valid theoretical explanation of tunneling started with the study of field-induced ionization of hydrogen atoms by Oppenheimer. ${ }^{1}$ His approach, in fact, formed the basis of the transfer Hamiltonian $(\mathrm{TH})$ method. The commonly referred formulation of the TH method is due to Bardeen. ${ }^{2}$ The essential idea of the TH method is the separation of space into two half spaces, each including one of the electrodes. Finding the solutions for the wave functions in these two half spaces, the transmission probability for the original problem can be calculated by using the first-order time-dependent perturbation theory. ${ }^{2,3}$ The Golden-rule-like expression for the transmission rate is given by

$$
P=\frac{2 \pi}{\hbar} \sum_{\mu, \nu}\left|M_{\mu \nu}\right|^{2} \delta\left(E_{\mu}-E_{\nu}\right),
$$

with the matrix element $M_{\mu \nu}$ defined as

$$
M_{\mu \nu}=-\frac{\hbar^{2}}{2 m} \int_{S_{0}}\left[\psi_{\mu}^{*} \nabla \psi_{\nu}-\psi_{\nu} \nabla \psi_{\mu}^{*}\right] \cdot d \mathbf{S},
$$

where $\psi_{\mu}$ and $\psi_{\nu}\left(E_{\mu}\right.$ and $\left.E_{\nu}\right)$ are the wave functions (eigenenergies) of the infinitely separated left and right electrode, respectively. The integration is carried out over surface $S_{0}$, which lies in the tunneling barrier, and is arbitrary otherwise. Despite its drawbacks discussed in the literature, the $\mathrm{TH}$ method is being widely used due to its simplicity and comprehensive transparency. For example, the theory of scanning tunneling microscopy (STM) as formulated by Tersoff and Hamann ${ }^{4}$ makes use of the TH method and yields a simple expression for tunneling current (for an $s$-wave tip state) in terms of the local density of states of the sample surface. The tunneling current, on the other hand, cannot be calculated using $a b$ initio methods due to immense computational requirements ${ }^{5}$. Consequently, the attention has been focused on modifying the original $\mathrm{TH}$ method in order to eliminate its shortcomings.

The perturbative nature of the $\mathrm{TH}$ method brings about certain restrictions on its applicability; it can only be used for high and thick barriers. However, there are two serious problems that the $\mathrm{TH}$ method faces in the context of STM. First, it is well established that the distance between the tip and the sample for the usual STM operation is small, ${ }^{6}$ and is in the range of $1-5 \AA$. For this situation, the first-order perturbation may not be sufficient to find the transmission probability, and multiple reflections from the boundaries of the tip and sample become important. Second, as a result of small separation, tip-sample interaction becomes important. Especially, the potential barrier in the vacuum gap is lowered due to the overlap of the charge densities of the two electrodes ${ }^{7}$ and the electronic structure of the electrodes is modified leading to the formation of tip-induced states. ${ }^{8}$ Consequently the wave functions for the infinitely separated electrodes $\psi_{\mu, \nu}$ are not appropriate to characterize the interacting tip-sample system.

In the following we examine whether the $\mathrm{TH}$ method can be modified in such a way as to eliminate the abovementioned problems. In Sec. II we study the effects of the boundary conditions for the wave functions and the position of the separation surface $S_{0}$ by using exactly solvable tunneling systems. ${ }^{9}$ We find that although it is possible to overcome some drawbacks of the original $\mathrm{TH}$ method, it is not possible to find a generally valid approximation. On the other hand, in Sec. III we show that by using Green's-function techniques, which include perturbations of all orders, it is possible to find the transmission probability exactly even in the presence of strong interelectrode interaction. An interesting result of the present work is that the position of the separation surface and boundary condition for the Green's function do 
not play any role as long as the transmission probability is concerned.

\section{THE MODIFIED TH METHOD}

Recently Chen ${ }^{10}$ proposed that the original $\mathrm{TH}$ method can be modified to calculate the transmission probability for low and thin barriers as well, even for cases for which the potential barrier is totally collapsed. Chen claimed that this can be achieved by using the wave functions $\bar{\psi}_{\mu}$ and $\bar{\psi}_{\nu}$ (instead of $\psi_{\mu}$ and $\psi_{\nu}$ ) for the infinitely separated electrodes, which takes the lowering of the barrier into account. To visualize the conjecture of Chen, the corresponding one-dimensional (1D) square barrier problem is depicted in Fig. 1. The height of the surface barrier for the infinitely separated electrodes is $V_{0}$ (the work function), and as a result of interelectrode interaction it is reduced to $V$ for the barrier thickness $d$. In the original TH method, $\psi_{\mu, \nu}$ are found by using the potential profiles shown in Fig. 1(b). Chen proposed that in the semi-infinite vacuum the potential has to be taken to be equal to $V_{0}$, and not to $V$, as in Fig. 1(c), to find $\bar{\psi}_{\mu, \nu}$. He calculated the matrix element $\bar{M}_{\mu \nu}$ in terms of these wave functions as in Eq. (2). He also showed that the second-order perturbation correction to Eq. (1) vanishes for this choice of potentials for the left and right half spaces.

First we derive the TH expression for the transmission probability of the 1D square barrier. To do so, one has to calculate the wave functions for the left and right half

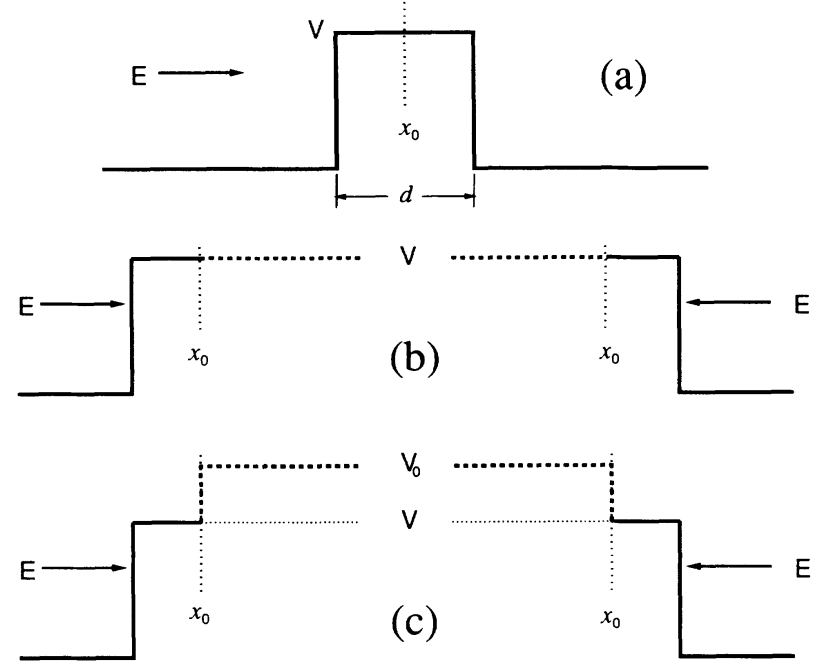

FIG. 1. Potential profile for (a) the 1D square barrier problem, and left and right half-spaces in (b) the original TH method and (c) Chen's conjecture.

spaces for the potentials shown in Figs. 1(b) and 1(c), and calculate the matrix element by using Eq. (2). The transmission rate calculated from Eq. (1) is normalized per unit incident flux to find the transmission probability. The final expression for the transmission probability according to Chen's conjecture is ${ }^{10}$

$$
T=\frac{16 \kappa_{0}^{2} k^{2}}{\left\{k^{2}\left(\cosh \kappa d / 2+\left[\kappa_{0} / \kappa\right] \sinh \kappa d / 2\right)^{2}+\kappa^{2}\left(\sinh \kappa d / 2+\left[\kappa_{0} / \kappa\right] \cosh \kappa d / 2\right)^{2}\right\}^{2}}
$$

with the wave vector $k=(2 m E)^{1 / 2} / \hbar$, inverse decay lengths $\kappa=[2 m(V-E)]^{1 / 2} / \hbar, \kappa_{0}=\left[2 m\left(V_{0}-E\right)\right]^{1 / 2} / \hbar$, and the separation point is taken to be midway between the electrodes at $x_{0}=d / 2$. The result for the original $\mathrm{TH}$ method can be retrieved by taking $\kappa_{o}=\kappa$. On the other hand, the transmission probability can be calculated exactly for the potential shown in Fig. 1(a) and is given by

$$
T=\frac{4 \kappa^{2} k^{2}}{4 \kappa^{2} k^{2}+\left(\kappa^{2}+k^{2}\right)^{2} \sinh ^{2} \kappa d} .
$$

In Fig. 2 the exact transmission probability is compared with those calculated by using the $\mathrm{TH}$ method. The original TH method yields a vanishing $T$ as $\kappa \rightarrow 0$, since the derivative of the wave functions $\psi_{\mu, \nu}$ vanishes. Thus, the original $\mathrm{TH}$ method cannot be used for low barrier heights. On the other hand, Chen's expression has a nonsingular behavior for $\kappa=0$ due to proper behavior of $\bar{\psi}_{\mu, \nu}$. For large barrier heights one observes that the transmission probability found by using the $\mathrm{TH}$ method is approximately equal to the exact result. However, for low barrier heights (i.e., $V \lesssim 0$ ) the mismatch between the exact result and that of the TH method as modified by Chen is more than $10 \%$. More importantly, for further

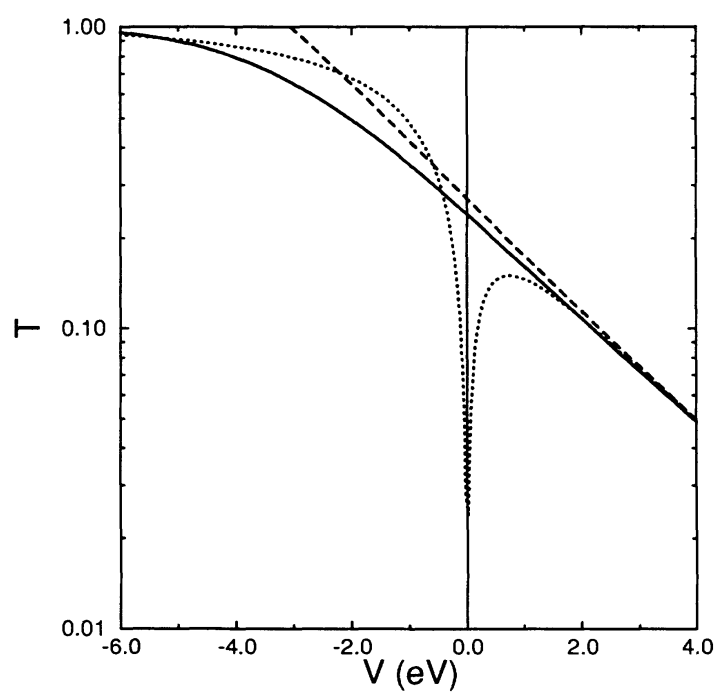

FIG. 2. Transmission probability for the 1D square barrier shown in Fig. 1. The full line is the exact result [Eq. (4)], and the dotted and dashed lines are the results of the original TH method and Chen's conjecture [Eq. (3)], respectively. The parameters are $d=2 \AA, E=12 \mathrm{eV}$, and $V_{0}=4 \mathrm{eV}$. 
lowering of the barrier (in our example shown in Fig. 2 for $V \lesssim-3 \mathrm{eV}$ ) transmission probability found by using Chen's conjecture exceeds unity, which is clearly unphysical. That is, although the singularity that $T$ attains for $V=0$ in the original $\mathrm{TH}$ method can be removed by using Chen's conjecture, the modified TH method still fails to give approximate results for vanishing and negative barrier heights.

Next we question the choice of Chen for the semiinfinite vacuum potential. Although for STM the potential outside the electrodes approaches $V_{0}$ away from the apex of the tip, for other tunneling systems (e.g., for semiconductor heterostructures) the vacuum level may not have the same physical significance. From this point of view this particular choice of boundary condition for the wave functions $\bar{\psi}_{\mu, \nu}$ cannot be justified. Therefore, we use $\kappa_{0}$ in Eq. (3) [or $V_{0}$ in Fig 1(c)] as a variable and calculate the corresponding transmission probability. The results are shown in Fig. 3(a) for different choices of $\kappa$. As can be observed in Eq. (3), $T \rightarrow 0$ as $\kappa_{0} \rightarrow 0$ or $\infty$. For finite values of $\kappa_{0}, T$ has a broad maximum. For high barrier heights the value of this maximum is very close to the exact $T$, thus any change in $V_{0}$ does not affect the transmission probability appreciably in accordance with the original $\mathrm{TH}$ method. On the other hand, for $V \lesssim 0$ the maximum becomes narrower and the boundary conditions specified for $\bar{\psi}_{\mu, \nu}$ at $x=x_{0}$ may lead to major variations in $T$ values.

Another crucial point in the $\mathrm{TH}$ method is the choice for the separation surface. In the original $\mathrm{TH}$ method, ${ }^{2}$ the transmission probability turns out to be independent of $x_{0}$ for high and thick barriers since the Wronskian of the wave functions is constant. This is also true for the $1 \mathrm{D}$ square barrier, which can be seen from Eq. (3) with $\kappa_{0}=\kappa$. However, Chen's conjecture yields an $x_{0^{-}}$ dependent $T$ as a result of changes in $\bar{\psi}_{\mu, \nu}$ for different boundary conditions. The dependence of the transmission probability on the position of the separation surface is shown in Fig. 3(b). Similar to Fig. 3(a), the transmission probability for high barrier heights is almost independent of $x_{0}$ as in the original TH method. For $V \lesssim 0$,
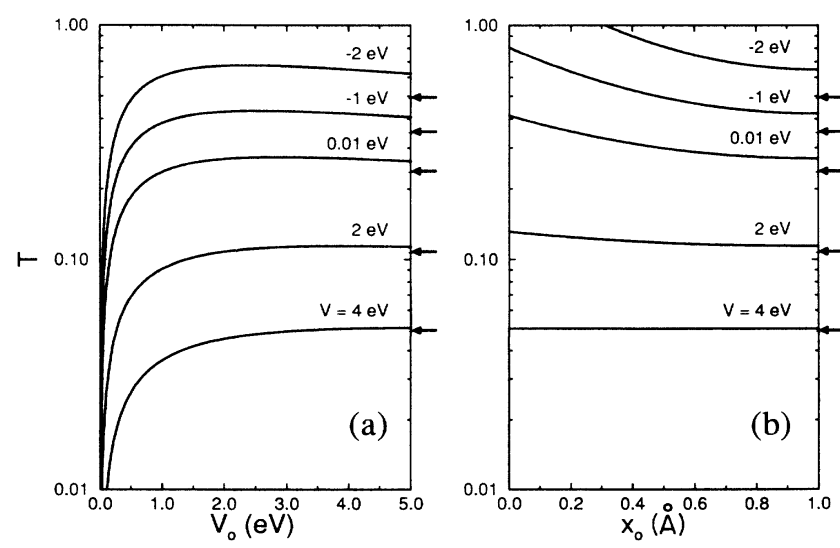

FIG. 3. Transmission probability calculated according to Chern's conjecture by varying (a) $V_{0}$ and (b) $x_{0}$ in Fig. 1(c). The curves are for $V=-2,-1,0.01,2$, and $4 \mathrm{eV}$ in the order of decreasing $T$. The arrows indicate the exact values of $T$.

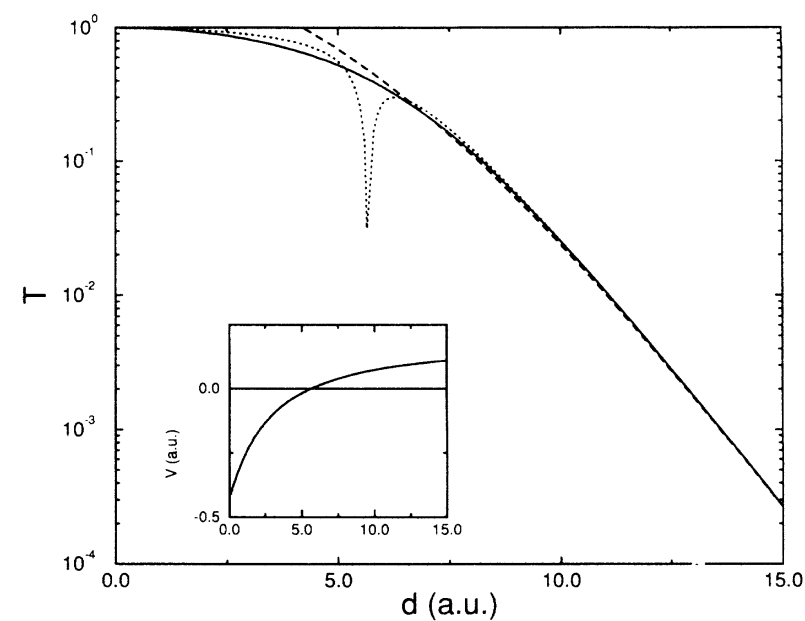

FIG. 4. Transmission probability for the bimetallic jellium potential. The curves are the same as in Fig. 2. Inset shows the variation of the maximum of the barrier height as a function of distance between the jellium edges. The jellium parameters are chosen to represent $\mathrm{Al}$.

however, $T$ rapidly increases as the separation surface gets closer to one of the electrodes. Note that the choice of the boundary conditions and separation surface may be important especially for asymmetrical barrier potentials, for which it is not possible (but necessary) to determine the separation surface and boundary conditions a priori. However, the $\mathrm{TH}$ method does not seem to be reliable from this respect.

We also use a bimetallic junction potential in order to test the validity of the above results for more realistic tunneling systems (see Ref. 9). To do so we use the local-density-overlapping charge-densities approximation of Smith and co-workers ${ }^{11}$. The solution for the transmission probability and wave functions $\psi_{\mu, \nu}$ and $\bar{\psi}_{\mu, \nu}$ is obtained by approximating the potential by a histogram profile and employing the transfer-matrix method. ${ }^{12}$ Ciraci and co-workers ${ }^{13}$ showed that this approximation gives reliable results for STM at small distances with blunt tips. Our results for transmission probability $T$ as a function of the interelectrode separation $d$ are shown in Fig. 4. The results are similar to those presented in Fig. 2, i.e., the original $\mathrm{TH}$ method leads to vanishing $T$ for $V=0$ and Chen's conjecture removes this singularity. An important observation is that for negative barrier heights (i.e., $V<0$ ) the original $\mathrm{TH}$ method, albeit qualitatively incorrect, gives a better quantitative approximation as compared to Chen's conjecture. This results from the unphysical behavior of the latter described above. Therefore, we find that for 1D problems under consideration the modified $\mathrm{TH}$ method as proposed by $\mathrm{Chen}^{10}$ (in terms of wave functions of the infinitely separated electrodes) is not better than the original $\mathrm{TH}$ method, and it does not yield admissible results for vanishing barrier heights.

\section{GREEN'S-FUNCTION TECHNIQUES}

In this section we focus our attention on Green'sfunction techniques proposed for the tunneling problem 
in conjunction with the $\mathrm{TH}$ method. In fact the methods described below are nonperturbative in nature since perturbation is extended to all orders. However, the underlying motivation is the same as that of the original $\mathrm{TH}$ method. Thus, we examine them in terms of their $\mathrm{TH}$ character. The Green's-function technique was applied to the tunneling problem by Caroli and co-workers. ${ }^{14}$ They started with two uncoupled electrodes at thermal equilibrium with different chemical potentials and introduced tunneling between them by using the Green'sfunction technique for nonequilibrium processes as formulated by Keldysh. ${ }^{15}$ They initially considered discrete systems, and later proposed a method to find the corresponding continuum limit. Feuchtwang ${ }^{16}$ formulated an alternative method starting from the boundary condition satisfied by the Green's functions at the separation surface and using the Keldysh method. Furthermore, he showed that the result of Caroli et al. can be obtained by using Green's functions vanishing at the interface in the framework of his method. He claimed, however, that the physically correct solution is found by using Green's functions with vanishing derivative at the interface. Recently Noguera ${ }^{17}$ questioned the physical significance of the boundary conditions used by both Caroli et al. ${ }^{14}$ and Feuchtwang. ${ }^{16}$ Using the matching procedure for the Green's functions at the separation surface, ${ }^{18}$ she found that the relevant quantity is the logarithmic derivative of the Green's function at the boundary. She expressed the transmission probability in terms of the logarithmic derivative of the Green's functions and showed that this expression reduces to those found by the others ${ }^{14,16}$ when specific boundary conditions are set. The tunneling current for STM has been calculated for specific geometries by using the original TH method, ${ }^{19}$ the method of Caroli et al. ${ }^{20}$ that of Noguera, ${ }^{21}$ and also by direct solution of the Schrödinger equation. ${ }^{22}$ Thus, a critical evaluation of Green's-function methods is important for making a comparison between different approaches. In what follows we show that the boundary condition specified at the separation surface for the Green's functions of the infinitely separated electrodes does not affect the transmission probability and all the above-cited approaches give the same result.

The Green's-function approach (in the TH context) relies on the determination of two Green's functions $G_{L}$ and $G_{R}$ for the left and right half spaces, respectively. The behavior of these Green's functions in the other half space is immaterial and only the boundary condition at the separation surface is relevant. For 1D systems the boundary condition used by Caroli et al. ${ }^{14}$ reads

$$
G_{L}^{C}\left(x_{0}, x^{\prime}<x_{0}\right)=0
$$

and that of Feuchtwang ${ }^{16}$ reads

$$
\left.\frac{\partial}{\partial x} G_{L}^{F}\left(x, x^{\prime}<x_{0}\right)\right|_{x=x_{0}}=0
$$

for the left half space, with similar expressions for the right half space. Clearly, the Green's function satisfying the boundary condition

$$
a G_{L}\left(x_{0}, x^{\prime}<x_{0}\right)+\left.b \frac{\partial}{\partial x} G_{L}\left(x, x^{\prime}\right)\right|_{x=x_{0}}=0
$$

can be written as

$$
G_{L}\left(x, x^{\prime}\right)=\alpha(a, b) G_{L}^{C}\left(x, x^{\prime}\right)+\beta(a, b) G_{L}^{F}\left(x, x^{\prime}\right) .
$$

Note that the logarithmic derivatives of $G_{L}$ and $G_{R}$ at $x_{0}$ are defined as

$$
\begin{aligned}
& X_{L}=-\left.\frac{\frac{\partial}{\partial x} G_{L}\left(x, x_{0}\right)}{G_{L}\left(x, x_{0}\right)}\right|_{x=x_{0_{-}}}, \\
& X_{R}=\left.\frac{\frac{\partial}{\partial x} G_{R}\left(x, x_{0}\right)}{G_{R}\left(x, x_{0}\right)}\right|_{x=x_{0_{+}}},
\end{aligned}
$$

respectively. For $b \neq 0, X_{L}$ can be written as

$X_{L}=\frac{a}{b} \frac{\left.\frac{\partial}{\partial x} G_{L}^{C}\left(x, x_{0}\right)\right|_{x=x_{0}}}{\left.\frac{\partial}{\partial x} G_{L}^{C}\left(x, x_{0}\right)\right|_{x=x_{0_{+}}}}+\frac{2 m}{\hbar^{2}} \frac{1}{G_{L}^{F}\left(x_{0}, x_{0}\right)}$.

As the numerator of the first term on the right-hand side vanishes, ${ }^{23}$ one obtains $X_{L}=2 m / \hbar^{2} G_{L}^{F}\left(x_{0}, x_{0}\right)$. Consequently, the transmission probability expressions of Noguera ${ }^{17}$ (i.e., $a, b \neq 0$, arbitrary otherwise),

$$
T=4 \frac{\operatorname{Im}\left\{X_{L}\right\} \operatorname{Im}\left\{X_{R}\right\}}{\left|X_{L}+X_{R}\right|^{2}}
$$

and that of Feuchtwang ${ }^{16}$ (i.e., $a=0$ ),

$$
T=4 \frac{\operatorname{Im}\left\{G_{L}^{F}\left(x_{0}, x_{0}\right)\right\} \operatorname{Im}\left\{G_{R}^{F}\left(x_{0}, x_{0}\right)\right\}}{\left|G_{L}^{F}\left(x_{0}, x_{0}\right)+G_{R}^{F}\left(x_{0}, x_{0}\right)\right|^{2}},
$$

are identical. The limit $b \rightarrow 0$ for Eq. (7) leads to an indeterminate logarithmic derivative $X_{L}$, since both the numerator and denominator in Eq. (9a) vanish. Applying the L'Hospital rule (differentiating the numerator and denominator with respect to $x_{0}$ and then taking the limit $x \rightarrow x_{0}$ from the left) one finds (using Ref. 23)

$$
X_{L, R}=\left.\frac{\hbar^{2}}{2 m} \frac{\partial^{2}}{\partial x \partial x^{\prime}} G_{L, R}^{C}\left(x, x^{\prime}\right)\right|_{x, x^{\prime}=x_{0}},
$$

which gives the transmission probability as found by Caroli et al. ${ }^{14}$ (i.e., $b=0$ ),

$$
T=\left.4 \frac{\operatorname{Im}\left(\frac{\partial^{2}}{\partial x \partial x^{\prime}} G_{L}^{C}\left(x, x^{\prime}\right)\right) \operatorname{Im}\left(\frac{\partial^{2}}{\partial x \partial x} G_{R}^{C}\left(x, x^{\prime}\right)\right)}{\left|\frac{\partial^{2}}{\partial x \partial x} G_{L}^{C}\left(x, x^{\prime}\right)+\frac{\partial^{2}}{\partial x \partial x} G_{R}^{C}\left(x, x^{\prime}\right)\right|^{2}}\right|_{x, x^{\prime}=x_{0}}
$$

This completes the derivation of the equivalence of Eqs. (11), (12) and (14). That is, we find that the TH methods making use of the Green's functions yield exactly the same result for $T$ irrespective of the boundary condition satisfied by $G_{L, R}$ on the separation surface [i.e., independent of $a$ and $b$ in Eq. (7)]. Rephrasing, we find that the only relevant boundary conditions for $X_{L}$ and $X_{R}$ are the ones which are satisfied at $x \rightarrow \pm \infty$, that is, outgoing wave boundary conditions. Thus, $G_{L, R}$ may be 
chosen as the Green's function for the complete system with appropriate scattered waves. This way it is possible to use Green's functions $G_{L, R}$ which are well defined at all points in space.

The transmission probability expression Eq. (11) can be modified to have the form

$$
T=\frac{\mathcal{W}\left[G_{L}^{*}, G_{L}\right] \mathcal{W}\left[G_{R}, G_{R}^{*}\right]}{\left|\mathcal{W}\left[G_{L}, G_{R}\right]\right|^{2}}
$$

with $\mathcal{W}$ denoting the Wronskian. In Eq. (15) the coordinates of the Green's functions are taken to be $x^{\prime}=x_{0}$ and $x=x_{0_{-}}\left(x_{0_{+}}\right)$for $G_{L}\left(G_{R}\right)$. In fact, since both Green's functions satisfy the same second-order differential equation (the Schrödinger equation), the Wronskians in Eq. (15) are independent of the point at which they are calculated provided that $x<x^{\prime}\left(x>x^{\prime}\right)$ for $G_{L}\left(G_{R}\right)$. Thus, the transmission probability as calculated by using the Green's-function method is independent of the position $x_{0}$ of the separation point. Exploiting this fact and using the asymptotic forms of these Green's functions far away from the barrier (where we assume the potential is essentially constant) one finds that the transmission probability as given in Eq. (11) [or equivalently Eq. (15)] is exact. This, in fact, is the demonstration of the nonperturbative nature of the Green's-function method.

To exemplify the validity of the above results, we calculate the transmission probability for the 1D square barrier shown in Fig. 1(a) using the Green's-function technique. The logarithmic derivatives $X_{L, R}$ can be found for $x_{0}=d / 2 \mathrm{as}^{24}$

$$
X_{L, R}=-\kappa \frac{\kappa \sinh \kappa d / 2-i k \cosh \kappa d / 2}{\kappa \cosh \kappa d / 2-i k \sinh \kappa d / 2}
$$

which when substituted in Eq. (11) yields the exact transmission probability Eq. (4). This result is independent of the position of the separation point $x_{0}$ as can easily be shown. On the other hand, the Wronskians in Eq. (15) can be found as

$$
\begin{aligned}
\mathcal{W}\left[G_{L}^{*}, G_{L}\right]= & \frac{k}{\kappa}\left|f\left(x_{0}\right)\right|^{2}, \\
\mathcal{W}\left[G_{R}, G_{R}^{*}\right]= & \frac{k}{\kappa}\left|g\left(x_{0}\right)\right|^{2}, \\
\mathcal{W}\left[G_{L}, G_{R}\right]= & {\left[\frac{\kappa^{2}-k^{2}}{2 \kappa^{2}} \sinh \kappa d / 2\right.} \\
& \left.-i \frac{k}{\kappa} \cosh \kappa d / 2\right] f\left(x_{0}\right) g\left(x_{0}\right),
\end{aligned}
$$

where $f$ and $g$ are functions of $x^{\prime}$, but clearly cancel out when substituted in Eq. (15) and one obtains the exact transmission probability Eq. (4), as expected. Calculations using the bimetallic jellium potential show that the Green's-function result for the transmission probability Eq. (11) gives the exact result, independent of both the position of the separation surface and the boundary condition satisfied by the Green's function.
Up to this point we have avoided addressing the question of the relation between the tunneling probability and the local density of states (LDOS) of the surfaces. This relation has been an issue of controversy in the former studies. ${ }^{14,16,17}$ Expressing the tunneling current in terms of the LDOS is important especially for STM applications, since the LDOS can be calculated by using $a b$ initio methods. As mentioned above, however, only for large tip-sample separations may it be possible to find the tunneling current in terms of the LDOS. It has been well established that ${ }^{14,16,17}$ the LDOS depends on the boundary condition chosen at the separation surface. That is, the LDOS can be defined for the electrode in contact with a certain vacuum potential (e.g., the surface potential or the infinite wall potential). On the other hand, the transmission probability is a property of the complete system, that is, two electrodes interacting with each other. As a result, writing the transmission probability in terms of the LDOS of electrodes in contact with a certain vacuum potential, albeit formally feasible, is not conceptually appealing for strongly interacting systems. Therefore, we do not interpret the above results in terms of the LDOS of the electrodes and refer only to the Green's functions.

To conclude, we verified that the Green's-function approach, which takes into account perturbations of all orders, can be used to find the transmission probability exactly. This procedure, on the other hand, requires complete information on the states of the respective electrodes in the presence of interelectrode interactions. Therefore, from a computational point of view, it does not yield any simplifications over the solution of the complete system. It has also been proposed ${ }^{17}$ to use two separation surfaces instead of one, which yields a factorization of the transmission probability in terms of quantities depending on the bulk properties of the electrodes and another one representing transmission through the vacuum barrier. In such a formulation, however, the central entity is still the vacuum transmission term which depends on the properties of the electrodes as well. That is, in all Green's-function approaches the difficulty arises from the inclusion of interelectrode interactions to the effective one-electron potentials, independent of the details of the calculation scheme. Some other Green's-function techniques ${ }^{25,26}$ making use of the full Green's function of the complete system also give the exact result for the transmission probability. However, these cannot be classified within the TH methods and thus we did not consider them in the present study.

\section{ACKNOWLEDGMENTS}

The author wishes to acknowledge Professor A. Baratoff and Dr. J. C. Chen for helpful discussions and Professor S. Ciraci for stimulating comments and careful examination of the manuscript. This work was partially supported by the Joint Project Agreement between Bilkent University and IBM Zurich Research Laboratory. 
${ }^{1}$ J. R. Oppenheimer, Phys. Rev. 13, 66 (1928).

${ }^{2}$ J. Bardeen, Phys. Rev. Lett. 6, 57 (1961).

${ }^{3}$ C. B. Duke, Tunneling in Solids (Academic, New York, 1969).

${ }^{4}$ J. Tersoff and D. R. Hamann, Phys. Rev. Lett. 50, 1998 (1983).

${ }^{5}$ Only a very limited set of problems concerning transmission probabilities can be solved using $a b$ initio methods. For an example, see M. D. Stiles and D. R. Hamann, Phys. Rev. Lett. 66, 3179 (1991).

${ }^{6}$ U. Durig, J. K. Gimzewski, and D. W. Pohl, Phys. Rev. Lett. 65, 349 (1990).

${ }^{7}$ S. Ciraci, A. Baratoff, and I. P. Batra, Phys. Rev. B 41, 2763 (1990); 42, 7618 (1990).

${ }^{8}$ E. Tekman and S. Ciraci, Phys. Scr. 38, 486 (1988); Phys. Rev. B 40, 10286 (1989).

${ }^{9}$ Systems for which the Schrödinger equation can be solved directly (either analytically or numerically, without employing any perturbative or Green's-function approach) are referred to as exactly solvable. In this context, the transfer matrix method (Ref. 12) used in the later sections is taken to be exact.

${ }^{10}$ C. J. Chen, Mod. Phys. Lett. B 5, 107 (1991).

${ }^{11}$ J. R. Smith, Phys. Rev. 181, 522 (1963); J. Ferrante and J. R. Smith, Surf. Sci. 38, 77 (1973).

${ }^{12}$ E. O. Kane, in Tunneling Phenomena in Solids, edited by E. Burnstein and S. Lundqvist (Plenum, New York, 1969).

${ }^{13}$ S. Ciraci, E. Tekman, M. Gökçedağ, I. P. Batra, and A. Baratoff, Ultramicroscopy (to be published); S. Ciraci,
E. Tekman, A. Baratoff, and I. P. Batra (unpublished).

${ }^{14}$ C. Caroli, R. Combescot, P. Nozieres, and D. Saint-James, J. Phys. C 4, 916 (1971); C. Caroli, R. Combescot, D. Lederer, P. Nozieres, and D. Saint-James, ibid. 4, 2598 (1971); Phys. Rev. B 12, 3977 (1975).

${ }^{15}$ L. V. Keldysh, Zh. Eksp. Teor. Fiz. 47, 1515 (1964) [Sov. Phys. JETP 20, 1018 (1965)].

${ }^{16}$ T. E. Feuchtwang, Phys. Rev. B 10, 4121 (1974); 12, 3979 (1975).

${ }^{17}$ C. Noguera, J. Microsc. 152, 3 (1988); Phys. Rev. B 42, 1629 (1990).

${ }^{18}$ J. E. Inglesfield, J. Phys. C 4, L14 (1971).

${ }^{19}$ N. D. Lang, Phys. Rev. B 36, 8173 (1987).

${ }^{20}$ J. Ferrer, A. Martin-Rodero, and F. Flores, Phys. Rev. B 38, 10113 (1988).

${ }^{21}$ W. Sacks and C. Noguera, Phys. Rev. B 43, 11612 (1991).

${ }^{22}$ S. Ciraci and E. Tekman, Phys. Rev. B 40, 11969 (1989).

${ }^{23}$ For the Green's function satisfying the boundary condition Eq. (5), one has

$$
\left.\frac{\partial G_{L}^{C}\left(x, x_{0_{-}}\right)}{\partial x}\right|_{x=x_{0}}=-\left.\frac{\partial G_{L}^{C}\left(x, x_{0_{+}}\right)}{\partial x}\right|_{x=x_{0}}=\frac{2 m}{\hbar^{2}} .
$$

${ }^{24}$ W. S. Sacks and C. Noguera, J. Microsc. 152, 23 (1988).

${ }^{25}$ L. Brey, G. Platero, and C. Tejedor, Phys. Rev. B 38, 10507 (1988).

${ }^{26}$ E. Kopatzki, G. Doyen, D. Drakova, and R. Behm, J. Microsc. 152, 687 (1988); G. Doyen, E. Koetter, J. P. Vigneron, and M. Scheffler, Appl. Phys. 51, 281 (1991). 